\title{
Comparison of sterol and fatty acid profiles of chytrids and their hosts reveals trophic upgrading of nutritionally inadequate phytoplankton by fungal parasites
}

Mélanie Gerphagnon (1) https://orcid.org/0000-0002-3363-4355, Ramsy Agha (1) https://orcid.org/0000-0002-6109-4624, Dominik Martin-Creuzburg (1) https://orcid.org/0000-0002-4248-0730, Alexandre Bec (1) https://orcid.org/0000-00034353-3766, Fanny Perriere, Cecilia Rad-Menéndez, Claire M. M. Gachon 는 https://orcid.org/0000-0002-3702-7472, Justyna Wolinska (1) https://orcid.org/0000-0003-2913-2923

DOI

10.1111/1462-2920.14489

Original publication date

3 December 2018

Document version

Accepted version

Published in

Environmental Microbiology

Citation (Vancouver)

Gerphagnon M, Agha R, Martin-Creuzburg D, Bec A, Perriere F, Rad-Menéndez C, et al. Comparison of sterol and fatty acid profiles of chytrids and their hosts reveals trophic upgrading of nutritionally inadequate phytoplankton by fungal parasites. Environmental Microbiology. 2019;21(3):949-58. 


\section{Comparison of sterol and fatty acid profiles of chytrids and their hosts reveals trophic}

upgrading of nutritionally inadequate phytoplankton by fungal parasites

Mélanie Gerphagnon ${ }^{1, a^{*}}$, Ramsy Agha ${ }^{1, a}$, Dominik Martin-Creuzburg ${ }^{2}$, Alexandre Bec ${ }^{3}$, Fanny Perriere $^{3}$, Cecilia Rad-Menéndez ${ }^{4}$, Claire M.M. Gachon ${ }^{4}$, and Justyna Wolinska ${ }^{1,5}$

(1) Leibniz-Institute of Freshwater Ecology and Inland Fisheries (IGB), Germany

(2) Limnological Institute, University of Konstanz, Mainaustrasse 252, 78464 Konstanz, Germany

(3) Laboratoire Microorganismes : Génome et Environnement- Université Clermont Auvergne, France

(4) Culture Collection of Algae and Protozoa, Scottish Association for Marine Science, Scottish Marine Institute, UK

(5) Department of Biology, Chemistry, Pharmacy, Institute of Biology, Freie Universität, Germany

${ }^{\text {a }} \mathrm{MG}$ and RA contributed equally to this paper

*Corresponding author: Mélanie Gerphagnon; Leibniz-Institute of Freshwater Ecology and Inland Fisheries (IGB), Mueggelseedamm 301, 12587 Berlin, Germany; Tel: +33(0)142.759.006; melaniegerphagnon@ hotmail.fr

Running title (not more than 50 characters): Trophic upgrading by chytrids

\section{Originality-Significance Statement}

Chytrids are important fungal parasites of phytoplankton that establish a trophic link between inedible or poorly nutritious phytoplankton taxa and zooplankton consumers (mycoloop). By characterizing the lipid profiles of two contrasting phytoplankton taxa and their respective chytrid parasites, this work reveals a novel qualitative facet of the mycoloop. In addition to rendering carbon and essential lipids from otherwise inedible phytoplankton hosts available to zooplankton, chytrids can upgrade the carbon of their hosts by de novo production of essential sterols. Evidence of trophic upgrading by chytrid parasites, together with their role as conveyors of carbon from inedible sources, emphasizes the importance of chytrid infection in enhancing carbon transfer in aquatic food webs. 


\section{Summary}

Chytrids are ubiquitous fungal parasites in aquatic ecosystems, infecting representatives of all major phytoplankton groups. They repack carbon from inedible phytoplankton hosts into easily ingested chytrid propagules (zoospores), rendering this carbon accessible to zooplankton. Grazing on zoospores may circumvent bottlenecks in carbon transfer imposed by the dominance of inedible or poorly nutritious phytoplankton (mycoloop). We explored qualitative aspects of the mycoloop by analyzing lipid profiles (fatty acids, sterols) of two chytrids infecting two major bloom-forming phytoplankton taxa of contrasting nutritional value: the diatom Asterionella formosa and the filamentous cyanobacterium Planktothrix agardhii. The polyunsaturated fatty acid composition of chytrids largely reflected that of their hosts, highlighting their role as conveyors of otherwise inaccessible essential lipids to higher trophic levels. We also showed that chytrids are capable of synthesizing sterols, thus providing a source of these essential nutrients for grazers even when sterols are absent in their phytoplankton hosts. Our findings reveal novel qualitative facets of the mycoloop, showing that parasitic chytrids, in addition to making carbon and essential lipids available from inedible sources, also upgrade their host's biochemical composition by producing sterols de novo, thereby enhancing carbon and energy fluxes in aquatic food webs.

\section{Introduction}

The trophic transfer efficiency across the autotroph-herbivore interface is crucial for carbon and energy fluxes in food webs. In aquatic ecosystems, phytoplankton communities are often dominated by species high resistance strategies to zooplankton grazing, such as large colonial diatoms or cyanobacteria (Ibelings et al., 2011; Perga et al., 2013; Rollwagen-Bollens et al., 2013). Reduced grazing may result in trophic bottlenecks, limiting the efficient transfer of carbon and energy to higher trophic levels.

Several phytoplankton species, including large taxa, can be infected by fungal parasites belonging to the phylum Chytridiomycota (i.e. chytrids). These true fungi are commonly found in freshwater, brackish, and marine pelagic environments (Lepère et al., 2016; Hassett et al., 2017; Maier and Peterson, 2017). Chytrid fungi display a characteristic free-living life stage as 
flagellated zoospores. Zoospores actively swim to find and infect new hosts. Upon encystment on a host, attached zoospores develop into a sporangium, a sessile reproductive structure which, once mature, releases new zoospores. In lakes, zoospore densities can reach more than 500 zoospores per $\mathrm{ml}$ (Jobard et al., 2010) and the infection prevalence can be extremely high during chytrid epidemics (>90\% of host cells are infected by at least one chytrid), as has been shown for filamentous cyanobacteria (Rasconi et al., 2012) and diatom populations (Ibellings et al., 2011). Zooplankton can take up chytrids by feeding on sporangia attached to edible hosts, i.e. concomitant predation. When phytoplankton assemblages are dominated by large inedible algae, zooplankton can still benefit from chytrid infections by efficiently feeding on zoospores. Zoospores have been proposed to constitute an alternative trophic link between primary and secondary production, the so-called mycoloop (Kagami et al., 2014). Experimental evidence shows that the mycoloop can sustain zooplankton somatic growth and reproduction when large inedible diatoms or poorly edible filamentous cyanobacteria dominate phytoplankton communities (Kagami et al., 2007; Agha et al., 2016). Food web models support the importance of chytrid infections in enhancing carbon and energy flows; chytrid parasitism may convey as much as $20 \%$ of gross primary production (in terms of carbon) and provide up to $38 \%$ of zooplankton dietary requirements (Grami et al. 2011; Rasconi et al., 2014; Haraldsson et al., 2018).

In addition to the quantitative importance of the mycoloop, chytrids might constitute a highquality food for zooplankton, being a source of essential lipids. Crustacean zooplankton does not synthesize polyunsaturated fatty acids (PUFA) and sterols de novo and thus need to acquire these essential lipids from their diet (Müller-Navarra et al., 2000; Martin-Creuzburg et al., 2008). PUFA are structural components of cell membranes and are involved in the modulation of a variety of vital membrane properties (Valentine and Valentine, 2004). Sterols, such as cholesterol, the predominant sterol in animals, are indispensable components of eukaryotic cell membranes and serve as precursors of steroid hormones (Goad, 1981). Due to the crucial importance of PUFA and sterols to consumers, de novo production of essential lipids by chytrid parasites might alleviate lipid deficiency in zooplankton, especially when phytoplankton communities are dominated by taxa lacking essential lipids. Cyanobacteria, as prokaryotes, differ from eukaryotic algae by lacking essential lipids, i.e. sterols and long-chain PUFA. Thus, during cyanobacterial blooms zooplankton may suffer from a deficiency in essential lipids, resulting in reduced growth and reproduction (Von Elert et al., 2003; Martin-Creuzburg et al., 2008). Chytrid infections might alleviate such deficiency if chytrid zoospores and/or sporangia 
produce essential lipids de novo. This mechanism might enhance overall trophic transfer efficiency in the pelagic food web. Chytrids have been shown to upgrade allochthonous organic matter by increasing digestibility of pollen grains and facilitating the transfer of otherwise inaccessible PUFA from pollen to metazoans through chytrid zoospores (Masclaux et al., 2013; Kagami et al., 2017). However, it is unclear if a similar biochemical upgrading of autochthonous carbon sources, such as phytoplankton, occurs upon infection by chytrid parasites. Although the characterization of the single species Zygorhizidium planktonicum, infecting the diatom Asterionella formosa, revealed that zoospores contain significant amounts of sterols (Kagami et al., 2007), it is still uncertain if chytrids incorporate sterols and other essential nutrients from their hosts, or if they synthesize them de novo.

The present study explores qualitative aspects of the mycoloop. Since phytoplankton taxa are highly diverse in terms of lipid composition, the influence of host lipid composition on chytrid zoospores might shape the functional importance of the mycoloop. We analyzed the lipid profiles of two phytoplankton hosts with contrasting nutritional quality for zooplankton (the long-chain PUFA- and sterol-rich diatom Asterionella formosa and the long-chain PUFA- and sterol-free cyanobacterium Planktothrix agardhii) and their respective chytrid parasites. We aimed to evaluate i) whether chytrids reflect the lipid composition of their hosts and thereby merely act as conveyors of inaccessible carbon and lipids to zooplankton consumers, and/or ii) if chytrids produce essential lipids de novo and therefore upgrade the carbon supplied by their hosts.

\section{Results}

\section{Carbon content, infected and uninfected hosts and parasites densities}

Carbon contents of uninfected host cells and zoospores were determined from carbon analyses and counts for each host-parasite system. Carbon contents were higher in uninfected A. formosa $\left(40.1 \pm 3.0 \mathrm{pg} \mathrm{C}\right.$ cell $\left.^{-1}\right)$ than in P. agardhii $\left(16.5 \pm 4.9 \mathrm{pg} \mathrm{C}\right.$ cell $\left.^{-1}\right)$. Zoospores infecting the diatom had three times lower carbon contents than zoospores infecting the cyanobacterium $\left(19.4 \pm 1.9 \mathrm{pg} \mathrm{C}\right.$ cell $^{-1}$ and $49.6 \pm 0.8 \mathrm{pg} \mathrm{C}$ cell $^{-1}$, respectively). Parasite-free host cultures had densities of $5.4 \pm 0.6 \times 10^{5}$ and $7.9 \pm 2.5 \times 10^{5}$ diatom and cyanobacteria cells $\mathrm{ml}^{-1}$, respectively. Infected diatom and cyanobacteria cultures were composed of $2.9 \pm 0.8 \times 10^{6}$ and $9.5 \pm 0.5 \times$ $10^{6}$ host cells, $4.5 \pm 1.5 \times 10^{5}$ and $2.6 \pm 1.9 \times 10^{5}$ attached sporangia as well as $6.6 \pm 0.3 \times 10^{3}$ and $2.8 \pm 0.3 \times 10^{4}$ zoospores $\mathrm{ml}^{-1}$, respectively. 


\section{$\underline{\text { Fatty acids }}$}

Uninfected hosts differed quantitatively and qualitatively in term of fatty acids (Fig. 2). While FA compositions of both hosts were similar in terms of major FA groups (Fig. 2B), they differed notably with regard to PUFA composition. The diatom displayed a diverse PUFA composition, characterized by the dominance of eicosapentaenoic acid (EPA; 20:5n-3; Fig. 2C; Table S1A), whereas the cyanobacterium did not contain any long-chain PUFA, but high concentrations of $\alpha$-linolenic acid (ALA, 18:3n-3) and linoleic acid (18:2n-6) instead (Fig 2D; Table S1B).

Infection by chytrid parasites increased total FA content in the Host+Sporangia fraction of the diatom system (about 5-fold), but not in the cyanobacterium system (Fig. 2A). A comparison of uninfected hosts and zoospores across both systems revealed quantitative and qualitative changes in FA compositions. Overall, chytrid zoospores were especially rich in FA (Fig. 2A; Table S1). A marked reduction in the proportion of PUFA was evident in zoospores of both systems, together with a general increase in monounsaturated fatty acids (MUFA; Fig. 2B). The latter were dominated by $16: 1 n-7$ and $18: 1 n-7$ in zoospores of both chytrid species, although 18:1n-7 was found at very low proportions and concentrations in their respective hosts (Fig. 2C, D, Table S1). Also, zoospores contained significant amounts of 18:1n-9 (Fig 2C, 2D, Table S1). Zoospores of both systems presented similar proportions of FA groups and total PUFA contents (Fig. 2B, Table S1). However, PUFA compositions of zoospores of both systems partially reflected that of their respective hosts. Specifically, the PUFA composition of the diatom-infecting chytrid was dominated by EPA, although other PUFA detected in the uninfected diatom were not detected in the chytrid zoospores (Fig 2C, Table S1). The cyanobacterium-infecting chytrid contained ALA and 18:2n-6 as the major PUFA, together with stearidonic acid (18:4n-3). The latter was not detected in the cyanobacterium and was putatively produced de novo (Fig. 2D).

Both systems presented significant differences in FA diversity (assessed by the ShannonWiener Index) among the three fractions analyzed: Uninfected Host, Host + Sporangia and Zoospores (Fig. S1, Table 1). Zoospores of the diatom-infecting chytrid displayed a significantly less diverse FA composition than two other fractions (Fig. S1, Table 1). The opposite was found in the cyanobacterium system, zoospores had more diverse FA profiles (Fig. S1, Table 1). 


\section{$\underline{\text { Sterols }}$}

The diatom A. formosa was rich in sterols, consisting mainly of epibrassicasterol and 22dehydrocholesterol (Fig. 3B), whereas the cyanobacterium $P$. agardhii lacked sterols completely (Fig. 3A).

Infection with chytrid parasites increased total sterol contents in both systems (Fig. 3A). In the diatom system, an increase in sterol diversity in the Host+Sporangia and Zoospores fractions was recorded, compared to the uninfected diatom (Fig. 3B, Fig S1). This was supported by significant differences in the Shannon-Wiener Index $\left(F_{2,3}=145.6 ; p<0.01\right.$, Table 1). In the cyanobacterium system, the Host+Sporangia fractions presented cholesterol and dihydrocholesterol, indicating de novo production by the chytrid parasite (Fig. 3B).

Zoospore fractions from both systems were characterized by high sterol content (Fig. 3A), with cholesterol as the most prominent sterol. Yet, diatom-infecting chytrid zoospores displayed a more diverse sterol profile than the cyanobacterium-infecting chytrid zoospores (Fig. 3B). In both cases, de novo sterol production by chytrid parasites was indicated by the detection of individual sterols in the Zoospores and Host+Sporangia fractions that were otherwise not detected in their respective Uninfected Host.

\section{Discussion}

It has been proposed that chytrids can circumvent trophic bottlenecks by repacking otherwise inaccessible phytoplankton carbon into readily ingestible zoospores, rendering it available to zooplankton (Kagami et al., 2007; Agha et al., 2016). In the present study, we investigated whether chytrids reflect the lipid composition of their phytoplankton hosts, and/or produce lipids de novo thus upgrading phytoplankton diet.

Carbon contents of both chytrid species are within the range of those reported earlier for another chytrid species, Zygorhizidium planktonicum (Kagami et al., 2007). We attribute differences in carbon contents between the two chytrid species used in our experiment to differences in the size of zoospores. Zoospores size has been shown to be a flexible parasite trait that varies with host genotype (Agha et al. 2018). As expected, zoospores of both chytrid species were rich in lipids, presenting a large lipid globule, a characteristic morphological feature of chytrids (Powell et al., 2015; Van den Wyngaert et al., 2018). Chytrids' high lipid contents could explain the observed increase in total FA contents in Host+Sporangia fraction compared to the uninfected diatom host. However, some fatty acids are known to exhibit anti- 
fungal activity (Lim et al., 2017) and this increase might also result from an immune response of host cells to infection.

Chytrids contained high proportions of MUFA, including 16:1n7, 18:1n7 and 18:1n9, which are common also in other fungi (Akinwole et al., 2014; Arce Funck et al., 2015). As MUFA are major substrates for $\beta$-oxidation (Bell et al., 2002; Cruz et al., 2018), they might be used by chytrid zoospores as endogenous lipid reserves to fuel swimming, as reported for aquatic zoosporic fungi belonging to the phylum Blastocladiomycota (Suberkropp \& Cantino 1973; Mills \& Cantino, 1977). Chytrid PUFA composition largely reflected that of their respective hosts. Like its host, the diatom-infecting chytrid contained almost exclusively EPA (20:5n-3), an important dietary constituent playing crucial role in zooplankton growth and reproduction (Müller-Navarra et al., 2000; Wacker and von Elert, 2001; Martin-Creuzburg et al., 2010). Also in line with its host, the cyanobacterium-infecting chytrid lacked EPA and contained linoleic acid (18:2n-6) and ALA (18:3n-3), in addition to stearidonic acid (18:4n-3) which was produced de novo. Despite the lack of EPA or other long-chain PUFA, this chytrid taxon arguably constitutes a source of essential PUFA for consumers, as zooplankton has been shown to use ALA and stearidonic acid to produce EPA, although this conversion is poorly efficient (Bec et al., 2003; Taipale et al., 2011). Marzetz et al. (2017) demonstrated the importance of dietary fatty acid diversity for Daphnia growth rates. Our results show that zoospores of the cyanobacterium-infecting chytrid presented the most diverse fatty acid profile, which suggests that this chytrid might biochemically upgrade the matter of its host. However, we found no evidence for a substantial qualitative or quantitative upgrading of host PUFA content or composition by chytrids.

The chytrids analyzed here largely reflected the FA compositions of their hosts. It is hence tempting to assume that contribution of chytrids to zooplankton PUFA demands will be determined by the phytoplankton species being infected. Yet, we cannot rule out that the observed differences in FA between two analysed chytrid species just reflect the phylogenetic distance (Akinwole et al., 2014). Cross-infection experiments and/or isotopic analyses could unequivocally show to what extent the host's chemical composition determines the nutritional quality of their chytrids parasites. Nonetheless, our study supports the idea that readily ingestible zoospores facilitate the transfer of PUFA from inedible or poorly edible phytoplankton taxa to zooplankton. Thus, the qualitative contribution of chytrids to PUFA demands of zooplankton seems to depend on which phytoplankton species is primarily infected. 
Arthropods are incapable of synthesizing sterols de novo and thus rely on dietary sources to cover their physiological demands (Von Elert et al., 2003; Wacker and MartinCreuzburg, 2012; Martin-Creuzburg et al., 2014). Our analyses show that the diatom A. formosa constitutes a potential source of sterols (mainly epibrassicasterol). However, transfer of sterols from large diatoms to zooplankton, such as the cladoceran Daphnia, is hampered by their lowingestibility (Kagami et al., 2007). In contrast, no sterols were detected in P. agardhii, supporting previous findings showing that cyanobacteria constitute a sterol-deficient food source for zooplankton (Von Elert et al., 2003; Martin-Creuzburg et al., 2008). Analyses of zoospores demonstrated de novo sterol biosynthesis. Chytrids infecting the sterol-free cyanobacterium contained significant amounts of cholesterol and dihydrocholesterol. Chytrid infecting diatoms contained epibrassicasterol in concentrations similar to what was found in the uninfected host but, in addition, synthesized some sterols de novo. Zoospores of this species presented the highest sterol diversity. Although no study has yet investigated the putative role of sterol diversity in trophic interactions, a positive impact of sterol diversity on Daphnia physiology cannot be ruled out, similar to the one demonstrated for fatty acid diversity (Marzetz et al., 2017). In spite of the phylogenetic distance, cholesterol was the predominant sterol in both chytrid species. The diatom-infecting chytrid, Zygorhizidium affluens, belongs to the order Lobulomycetales which is distantly related to other Chytridiomycota orders, including the here used cyanobacterium-infecting chytrid, Rhizophydium megarrhizum (order Rhizophydiales), (Rad-Menèndez et al., 2018). Cholesterol is also the main sterol in Zygorhizidium planktonicum (Kagami et al., 2007), another diatom-infecting chytrid belonging to the order Novel clade II (sensu Jobard et al., 2012), and in four other species belonging to the order Chytridiales (Weete et al., 2010). Taken together, cholesterol content seems to be a general feature of fungi of the phylum Chytridiomycota. Since cholesterol is the main sterol in most animals, and is often hardly represented in plants or algae (Martin-Creuzburg and Merkel, 2016), high cholesterol content in zoospores stresses the nutritional importance of chytrids for consumers. The qualitative contribution of the mycoloop to sustain secondary production would be even more significant when phytoplankton communities are dominated by inedible and/or sterol-deficient species, as chytrids produce sterols de novo, increase sterol diversity and concentrate existing sterols in readily ingestible zoospores.

\section{Conclusion}


Lipid analyses reported here uncover an additional facet of the mycoloop. The PUFA composition of chytrid parasites generally reflected that of their phytoplankton hosts. However, chytrids produced sterols de novo that are otherwise absent in their phytoplankton host. Therefore, in addition to conveying carbon from inedible or poorly edible sources in the form of readily ingestible zoospores, chytrid parasites i) convey essential fatty acids from their phytoplankton hosts to zooplankton and ii) upgrade the biochemical quality of host-derived organic matter by synthesizing sterols de novo. These findings stress the qualitative importance of the mycoloop as trophic link contributing to the coupling of primary and secondary production in pelagic food webs.

\section{Experimental Procedures}

\section{$\underline{\text { Host and parasite cultures }}$}

Two host-parasite systems were used in this study: 1) the diatom A. formosa, strain CCAP 1005/23, with its chytrid parasite Zygorhizidium affluens, strain CCAP4086/1 (both isolated from Lake Pavin, France, in 2013) and 2) the cyanobacterium P. agardhii, strain NIVACYA630 (isolated from Lake Lyseren, Norway, in 2008) with its chytrid parasite Rhizophydium megarrhizum, strain Chy-Kol2008 (isolated from Lake Kolbotnvatnet, Norway, in 2008). The diatom host was maintained as non-axenic batch cultures in DM medium (Beakes et al., 1988) at $20^{\circ} \mathrm{C}$ and a $12 / 12 \mathrm{~h} \mathrm{light/dark} \mathrm{regime}\left(64 \mu \mathrm{mol} \mathrm{m} \mathrm{m}^{-2} \mathrm{~s}^{-1}\right)$. Its chytrid parasite was maintained on its diatom host at $16^{\circ} \mathrm{C}$ and the same light conditions, by transferring $20 \%$ (vol/vol) of a synchronized and highly infected culture into an uninfected host culture every weeks. The cyanobacterium host was maintained in Z8 medium (Kotai, 1972) as non-axenic batch cultures at $16^{\circ} \mathrm{C}$ and constant irradiance $\left(15 \mu \mathrm{mol} \mathrm{m} \mathrm{m}^{-2} \mathrm{~s}^{-1}\right)$. The chytrid parasite was maintained on its cyanobacterium host cultivated at the same temperature and light conditions by transferring zoospore suspensions into uninfected cultures every two weeks (Sonstebo and Rohrlack, 2011). All culture transfers were performed aseptically. Cultures were mixed daily through gentle swirling by hand.

\section{Experimental set-up}

Uninfected cultures of A. formosa and P. agardhii were scaled up for 4 weeks and maintained as triplicate exponentially-growing semi-continuous cultures by weekly dilutions (1:2 in Z8 medium and 1:4 in DM medium, respectively). About 20\% volume of each replicated culture 
was separated whereas the remaining volume $(80 \%$ vol) was inoculated with their respective chytrid parasites. Uninfected and infected cultures were further incubated under the above conditions . One A. formosa replicate (infected and uninfected) was lost due to handling error. Eleven days after the addition of chytrids, advanced infection and the presence of freeswimming zoospores were confirmed microscopically and cultures were size-fractionated for the analysis of carbon, sterols, and fatty acids (Fig. 1). Briefly, infected cultures containing a mixture of uninfected hosts, infected hosts (with attached sporangia), free-swimming zoospores, and heterotrophic bacteria, were filtered successively through $25 \mu \mathrm{m}, 10 \mu \mathrm{m}$ and 5 $\mu \mathrm{m}$ sterile nylon meshes and a $3 \mu \mathrm{m}$ polycarbonate filter. The largest fraction (i.e. $>3 \mu \mathrm{m}$ ), containing infected (incl. attached sporangia) and uninfected host cells was collected by washing the successive meshes with sterile DM or Z8 medium (depending on the system) and transferring the concentrate into sterile Erlenmeyer flasks. The filtrate, i.e. the $<3 \mu \mathrm{m}$ fraction including zoospores and heterotrophic bacteria, was transferred to sterile glass Erlenmeyer flasks. Each size-fraction was then splitted into three sub-samples: one for particulate organic carbon (POC), one for fatty acid (FA), and one for sterol analyses (Fig.1). For FA and sterol analysis, each dedicated sub-sample was filtered on pre-combusted Whatman ${ }^{\mathrm{TM}} \mathrm{GF} / \mathrm{C}$ glassfiber filters $(\varnothing 47 \mathrm{~mm})$. Filters were kept at $-80^{\circ} \mathrm{C}$ until lipid analyses. POC was determined from the sub-sample of each fraction, loaded onto pre-combusted and pre weighted Whatman ${ }^{\mathrm{TM}}$ GF/C glass-fiber filters ( $\varnothing 25 \mathrm{~mm}$ ). The filters were dried for $24 \mathrm{~h}$, weighed, and analyzed by combustion elemental analysis using an Elementar Vario EL analyzer (Elementar, Germany).

Bacterial POC in the zoospore fraction $(<3-1.2 \mu \mathrm{m})$ was estimated from differences in bacterial counts (see below for counting methodology) before $(<3-1.2 \mu \mathrm{m})$ and after filtration through GF/C glass-fiber filters $(<1.2 \mu \mathrm{m}$; Fig.1), assuming an average carbon content of $20 \mathrm{fg}$ cell $^{-1}$ (Ducklow \& Carlson 1992).

Aliquots from each fraction of infected cultures were fixed i) in formaldehyde ( $2 \%$ final concentration) to determine zoospore and bacterial densities and ii) in Lugol's iodine solution to quantify host and sporangia densities (Fig.1). FA, sterols, and POC of the Uninfected hosts were analyzed accordingly, following the same procedures as above.

\section{Fatty acid analysis}

Filters containing biomass from the different fractions $(1.78-2.1 \mathrm{mgC}, 15.5-20.1 \mathrm{mgC}$ and 4.9 - 7.5 mgC for Zoospore, Host+Sporangia and Uninfected host fractions, respectively) were extracted twice using chloroform:methanol (2/1), following the method of Folch et al. (1957). FA in the total lipid extract were converted into FA methyl-esters (FAME) by acid-catalyzed 
transesterification after adding non-methylated 13:0 and 23:0 as internal standards according to a modified protocol of Christie $\left(1982 ; 4 \% \mathrm{H}_{2} \mathrm{SO}_{4}\right.$ in methanol at $75^{\circ} \mathrm{C}$ for $2 \mathrm{~h}$ ). FAME were analyzed on a gas chromatograph (Agilent technologies 6850, Santa Clara, CA, USA) equipped with a DB-Wax column (J\&W Scientific, $30 \mathrm{~m} \times 0.25 \mathrm{~mm}$ ID $\times 0.25 \mathrm{~mm}$ film), and a flame ionization detector $\left(250^{\circ} \mathrm{C}\right.$; split injection; carrier gas: helium; oven temperature ramp $150^{\circ} \mathrm{C}$ to $250^{\circ} \mathrm{C}$ at $\left.3^{\circ} \mathrm{C} \min ^{-1}\right)$. FAME were quantified against internal standards $\left(20 \mu \mathrm{g} \mathrm{sample}{ }^{-1}\right.$ of 13:0 and 23:0) and identified by comparing retention times with those obtained from Supelco® standards (37 Component FAME Mix), Larodan FAME standards, and their mass spectra which were recorded using a gas chromatograph-mass spectrometer (GC-MS; Agilent Technologies, 5975B MSD). FAME concentrations were then normalized by POC contents in respective samples.

\section{$\underline{\text { Sterol analysis }}$}

Filters prepared for sterol analysis were deposited in dichloromethane/methanol $(2: 1, \mathrm{v} / \mathrm{v})$ and stored at $-20^{\circ} \mathrm{C}$ overnight. Prior to extraction, $5 \alpha$-cholestane was added as internal standard (1 $\left.\mu \mathrm{g} \mathrm{sample}^{-1}\right)$. Total lipids were extracted twice with dichloromethane/methanol $(2: 1, \mathrm{v} / \mathrm{v})$. The pooled cell-free lipid extracts were evaporated to dryness under $\mathrm{N}_{2}$-atmosphere and saponified with methanolic $\mathrm{KOH}\left(0.2 \mathrm{~mol} \mathrm{~L}{ }^{-1}, 70^{\circ} \mathrm{C}, 1 \mathrm{~h}\right)$. Subsequently, neutral lipids were partitioned into iso-hexane:diethylether $(9: 1, \mathrm{v} / \mathrm{v})$, evaporated to dryness under $\mathrm{N}_{2}$ and resuspended in isohexane $(10-30 \mu \mathrm{l})$. Sterols were analyzed and quantified by gas chromatography (GC) using an HP 6890 gas chromatograph (Agilent Technologies, Santa Clara, CA, USA) equipped with a flame ionization detector (FID) and an HP-5 capillary column (Agilent Technologies, $30 \mathrm{~m} \times$ $0.25 \mathrm{~mm} \mathrm{ID} \times 0.25 \mathrm{~mm}$ film). Additional GC configurations are given elsewhere (MartinCreuzburg et al., 2016). The injection volume was $1 \mu$ l. Sterols were quantified by comparison to $5 \alpha$-cholestane using multipoint calibration curves generated for a set of sterols that are available commercially. Sterols for which authentic standards were not available were quantified as cholesterol equivalents using the cholesterol calibration curve; C-24 epimers and cis-trans isomers were quantified using the calibration curve of the respective epimer/isomer available as authentic standard. The limit for quantification of sterols was $10 \mathrm{ng}$. Sterols were identified based on their relative retention times and their mass spectra, which were recorded using a gas chromatograph-mass spectrometer (GC-MS; Agilent Technologies, 5975 C inert MSD) equipped with a fused-silica capillary column (HP-5MS, Agilent; GC settings as described above for FID). Sterols were analyzed both in their free form and as their trimethylsilyl derivatives, which were prepared by incubating $20 \mu \mathrm{l}$ of the iso-hexane sterol 
extracts with $10 \mu \mathrm{l}$ of $\mathrm{N}, \mathrm{O}$-bis(trimethylsilyl)trifluoroacetamide including $1 \%$ trimethylchlorosilane for $1 \mathrm{~h}$ at room temperature. Mass spectra were recorded between $\mathrm{m} / \mathrm{z} 50$ and 600 in the electron ionization mode, and then compared with mass spectra of authentic standards and mass spectra found in a self-generated spectra library. The C-24 stereochemistry could not be identified with certainty and thus was not further specified or, if possible, derived from literature data. For instance, the 24-methylcholesta-5,22-dien-3 $\beta$-ol detected in A. formosa was assumed to show the $24 \alpha$ configuration, thus representing epibrassicasterol rather than brassicasterol as has been proposed for other diatom species (Martin-Creuzburg and Merkel 2016). Sterol concentrations were related to the POC contents of their respective samples.

\section{$\underline{\text { Host, sporangia, zoospore and free-living bacteria densities }}$}

Host and sporangia fractions (>3 $\mathrm{mm}$ ). Host cells density (or filaments for cyanobacteria) and sporangia densities were estimated from 0.5 to $0.8 \mathrm{ml}$ of Lugol-fixed samples using a Sedgewick-Rafter chamber and a Nikon Ti Eclypse inverted microscope. At least 400 host cells (or filaments) were investigated per sample.

Zoospores fraction (3-1.2 $\mu \mathrm{m})$. Zoospore quantification was estimated from formaldehydefixed samples and analyzed using a Sedgewick-Rafter chamber. At least $20 \mu 1$ (or 150 zoospores) were counted per sample. To rule out host contamination, the entire chamber was checked but no host cells or filaments were found. Bacterial densities were determined using a haemocytometer and a Zeiss Axioskop epifluorescence microscope after staining with 4', 6diamidino-2-phenylindole (DAPI; $1 \mu \mathrm{g} \mathrm{ml}^{-1}$ final conc.) followed by $1 \mathrm{~h}$ incubation in the dark. Bacterial POC in the zoospores fraction accounted for $6.5 \pm 3.4 \%$ and $3.4 \pm 0.5 \%$ of total POC in the diatom and the cyanobacterial systems, respectively. Lipid concentrations were related to the zoospore biomass only.

\section{$\underline{\text { Data analysis }}$}

Fatty acid $\left(\mathrm{H}_{\mathrm{FA}}\right)$ and sterol $\left(\mathrm{H}_{\mathrm{ST}}\right)$ diversity within individual fractions was calculated using the Shannon-Wiener index. $\mathrm{H}_{\mathrm{FA}}$ calculation included the 27 fatty acids representing at least $1 \%$ of total FA in at least one fraction in each system. Because sterol diversity was low, no preselection was made to calculate sterol $\mathrm{H}_{\mathrm{ST}}$. One-way ANOVAs were performed for the $\mathrm{H}_{\mathrm{FA}}$ and $\mathrm{H}_{\mathrm{ST}}$ of each system, including the different fractions (i.e. Uninfected Host, Host+Sporangia and Zoospores) as fixed factors, followed by Tukey's post hoc tests. Data were not normally distributed, even after transformation. ANOVA was still applied as the $\mathrm{H}_{\mathrm{FA}}$ and $\mathrm{H}_{\mathrm{ST}}$ of both systems showed equal variances of the residuals (Bartlett test; $\alpha=0.05$ ). 
Q-q-plots of the residuals revealed no major deviations from normality. ANOVA, while very sensitive to lack of homocedasticity (which is not the case here) is very robust to deviations normality. Even moderate deviations from normality have little effect in false positive rates (Glass et al., 1972; Harwell et al., 1992; Lix et al., 1996).

\section{Acknowledgments}

We express our gratitude to Thomas Rohrlack, for supplying the cyanobacterium-infecting chytrid strain. We also would like to thank Alina Gross for her assistance. We want to thank the two anonymous reviewers for improving this manuscript with their valuable comments. We are especially grateful to one of the referees, who showed a level of engagement in the review process rarely seen by the authors. His/her valuable input has greatly contributed to the final version of the manuscript. MG was funded by the International IGB Fellowship Program "Freshwater Science". RA was supported by a postdoctoral fellowship from the Alexander von Humboldt Foundation.

\section{Conflict of interest}

The authors declare that they have no conflict of interest. 


\section{References}

Agha, R., Saebelfeld, M., Manthey, C., Rohrlack, T., and Wolinska, J. (2016) Chytrid parasitism facilitates trophic transfer between bloom-forming cyanobacteria and zooplankton (Daphnia). Sci Rep 6: 35039.

Agha, R., Gross, A., Gerphagnon, M., Rohrlack, T., \& Wolinska, J. (2018). Fitness and eco-physiological response of a chytrid fungal parasite infecting planktonic cyanobacteria to thermal and host genotype variation. Parasitology 145: 1279-1286

Akinwole, P.O., Lefevre, E., Powell, M.J., and Findlay, R.H. (2014) Unique odd-chain polyenoic phospholipid fatty acids present in chytrid Fungi. Lipids 49: 933-942.

Arce Funck, J.A., Bec, A., Perrière, F., Felten, V., and Danger, M. (2015) Aquatic hyphomycetes: a potential source of polyunsaturated fatty acids in detritus-based stream food webs. Fungal Ecol 13: 205-210.

Beakes, G.W., Canter, H.M., and Jaworski, G.H.M. (1988) Zoospore ultrastructure of Zygorhizidium affluens and Z. planktonicum, two chytrids parasitizing the diatom Asterionella formosa. Can J Bot 66: 1054-1067.

Bec, A., Desvilettes, C., Véra, A., Fontvieille, D., and Bourdier, G. (2003) Nutritional value of different food sources for the benthic Daphnidae Simocephalus vetulus: role of fatty acids. Archiv für Hydrobiologie 156: 145-163.

Bell, J.G., Henderson, R.J., Tocher, D.R., McGhee, F., Dick, J.R., Porter, A. et al. (2002) Substituting fish oil with crude palm oil in the diet of Atlantic salmon (Salmo salar) affects muscle fatty acid composition and hepatic fatty acid metabolism. J Nutr 132: 222-230.

Christie, W.W., and Han, X. (2012) Lipid extraction, storage and sample handling. In Lipid Analysis (Fourth Edition): Woodhead Publishing.

Cruz, M.M., Lopes, A.B., Crisma, A.R., de Sá, R.C., Kuwabara, W.M., Curi, R. et al. (2018) Palmitoleic acid (16: 1n7) increases oxygen consumption, fatty acid oxidation and ATP content in white adipocytes. Lipids Health Dis 17: 55.

Folch, J., Lees, M., and Sloane-Stanley, G. (1957) A simple method for the isolation and purification of total lipids from animal tissues. J Biol Chem 226: 497-509.

Harwell, M. R., Rubinstein, E. N., Hayes, W. S., \& Olds, C. C. (1992). Summarizing Monte Carlo results in methodological research: The one-and two-factor fixed effects ANOVA cases. J Educ Behav Stat 17: 315-339.

Glass, G. V., Peckham, P. D., \& Sanders, J. R. (1972). Consequences of failure to meet assumptions underlying the fixed effects analyses of variance and covariance. Rev Educ Res, 42: 237-288.

Goad, L. (1981) Sterol biosynthesis and metabolism in marine invertebrates. Pure Appl Chem 53: 837852.

Haraldsson, M., Gerphagnon, M., Bazin, P., Colombet, J., Tecchio, S., Sime-Ngando, T., and Niquil, N. (2018) Microbial parasites make cyanobacteria blooms less of a trophic dead end than commonly assumed. ISME J: 1008-1020.

Hassett, B.T., Ducluzeau, A.L.L., Collins, R.E., and Gradinger, R. (2017) Spatial distribution of aquatic marine fungi across the western Arctic and sub-arctic. Environ Microbiol 19: 475-484.

Ibelings, B.W., Gsell, A.S., Mooij, W.M., Van Donk, E., Van den Wyngaert, S., and De Senerpont Domis, L.N. (2011) Chytrid infections and diatom spring blooms: paradoxical effects of climate warming on fungal epidemics in lakes. Freshw Biol 56: 754-766.

Jobard, M., Rasconi, S., \& Sime-Ngando, T. (2010). Fluorescence in situ hybridization of uncultured zoosporic fungi: testing with clone-FISH and application to freshwater samples using CARD-FISH. J Microbiol Meth 83: 236-243.

Kagami, M., Miki, T., and Takimoto, G. (2014) Mycoloop: chytrids in aquatic food webs. Front Microbiol 5: 166.

Kagami, M., Motoki, Y., Masclaux, H., and Bec, A. (2017) Carbon and nutrients of indigestible pollen are transferred to zooplankton by chytrid fungi. Freshw Biol 62: 954-964. 
Kagami, M., Von Elert, E., Ibelings, B.W., De Bruin, A., and Van Donk, E. (2007) The parasitic chytrid, Zygorhizidium, facilitates the growth of the cladoceran zooplankter, Daphnia, in cultures of the inedible alga, Asterionella. Proc R Soc Lond B Biol Sci 274: 1561-1566.

Kotai, J. (1972) Instructions for preparation of modified nutrient solution Z8 for algae. Norwegian Institute for Water Research, Oslo 11: 5.

Lepère, C., Ostrowski, M., Hartmann, M., Zubkov, M.V., and Scanlan, D.J. (2016) In situ associations between marine photosynthetic picoeukaryotes and potential parasites-a role for fungi? Environ Microbiol Rep 8: 445-451.

Lim, G. H., Singhal, R., Kachroo, A., \& Kachroo, P. (2017). Fatty acid-and lipid-mediated signaling in plant defense. Annu Rev Phytopathol, 55: 505-536.

Lix, L. M., Keselman, J. C., \& Keselman, H. J. (1996). Consequences of assumption violations revisited:

A quantitative review of alternatives to the one-way analysis of variance $\mathrm{F}$ test. Rev Educ Res, 66:

579-619.

Maier, M.A., and Peterson, T.D. (2017) Prevalence of chytrid parasitism among diatom populations in the lower Columbia River Freshw Biol 62: 414-428.

Martin-Creuzburg, D., and Merkel, P. (2016) Sterols of freshwater microalgae: potential implications for zooplankton nutrition. J Plankton Res 38: 865-877.

Martin-Creuzburg, D., von Elert, E., and Hoffmann, K.H. (2008) Nutritional constraints at the cyanobacteria-Daphnia magna interface: The role of sterols. Limnol Oceanogr 53: 456-468.

Martin-Creuzburg, D., Wacker, A., and Basena, T. (2010) Interactions between limiting nutrients: consequences for somatic and population growth of Daphnia magna. Limnol Oceanogr 55: 25972607.

Martin-Creuzburg, D., Oexle, S., and Wacker, A. (2014) Thresholds for sterol-limited growth of Daphnia magna: a comparative approach using 10 different sterols. J Chem Ecol 40: 1039-1050.

Marzetz, V., Koussoroplis, A.-M., Martin-Creuzburg, D., Striebel, M., and Wacker, A. (2017) Linking primary producer diversity and food quality effects on herbivores: A biochemical perspective. Sci Rep 7: 11035.

Masclaux, H., Perga, M.-E., Kagami, M., Desvilettes, C., Bourdier, G., and Bec, A. (2013) How pollen organic matter enters freshwater food webs. Limnol Oceanogr 58: 1185-1195.

Mills \& Cantino, 1977, Isolation and characterization of lipid globules from the zoospores of Blastocladiella emersonii. Arch of Microbiol, 112: 195-200.

Müller-Navarra, D.C., Brett, M.T., Liston, A.M., and Goldman, C.R. (2000) A highly unsaturated fatty acid predicts carbon transfer between primary producers and consumers. Nature 403: 74-77.

Perga, M.-E., Domaizon, I., Guillard, J., Hamelet, V., and Anneville, O. (2013) Are cyanobacterial blooms trophic dead ends? Oecologia 172: 551-562.

Powell, M.J., Letcher, P.M., Chambers, J.G., and Roychoudhury, S. (2015) A new genus and family for the misclassified chytrid, Rhizophlyctis harderi. Mycologia 107: 419-431.

Rad-Menéndez, C., Gerphagnon, M., Garvetto, A., Arce, P., Badis, Y., Sime-Ngando, T., \& Gachon, C. M. (2018). Rediscovering Zygorhizidium affluens Canter - Molecular taxonomy, infectious cycle, and cryopreservation of a chytrid infecting the bloom-forming diatom Asterionella formosa. Appl Environ Microbiol, AEM-01826.

Rasconi, S., Niquil, N., \& Sime-Ngando, T. (2012). Phytoplankton chytridiomycosis: community structure and infectivity of fungal parasites in aquatic ecosystems. Environ Microbiol, 14: 21512170.

Rasconi, S., Grami, B., Niquil, N., Jobard, M., and Sime-Ngando, T. (2014) Parasitic chytrids sustain zooplankton growth during inedible algal bloom. Front Microbiol $\mathbf{5}$.

Rollwagen-Bollens, G., Bollens, S.M., Gonzalez, A., Zimmerman, J., Lee, T., and Emerson, J. (2013) Feeding dynamics of the copepod Diacyclops thomasi before, during and following filamentous cyanobacteria blooms in a large, shallow temperate lake. Hydrobiologia 705: 101-118. 
Sonstebo, J.H., and Rohrlack, T. (2011) Possible implications of chytrid parasitism for population subdivision in freshwater cyanobacteria of the genus Planktothrix. Appl Environ Microbiol 77: 13441351.

Suberkropp \& Cantino 1973, Utilization of endogenous reserves by swimming zoospores of Blastocladiella emersonii, Arch of Microbiol, 89: 205-221.

Taipale, S.J., Kainz, M.J., and Brett, M.T. (2011) Diet-switching experiments show rapid accumulation and preferential retention of highly unsaturated fatty acids in Daphnia. Oikos 120: 1674-1682.

Valentine, R.C., and Valentine, D.L. (2004) Omega-3 fatty acids in cellular membranes: a unified concept. Prog Lipid Res 43: 383-402.

Van den Wyngaert, S., Rojas-Jimenez, K., Seto, K., Kagami, M., and Grossart, H.P. (2018) Diversity and hidden host specificity of chytrids infecting colonial volvocacean algae. J Eukaryot Microbiol.

Von Elert, E., Martin-Creuzburg, D., and Le Coz, J.R. (2003) Absence of sterols constrains carbon transfer between cyanobacteria and a freshwater herbivore (Daphnia galeata). Proc $R$ Soc Lond $B$ Biol Sci 270: 1209-1214.

Wacker, A., and von Elert, E. (2001) Polyunsaturated fatty acids: evidence for non-substitutable biochemical resources in Daphnia galeata. Ecology 82: 2507-2520.

Wacker, A., and Martin-Creuzburg, D. (2012) Biochemical nutrient requirements of the rotifer Brachionus calyciflorus: co-limitation by sterols and amino acids. Funct Ecol 26: 1135-1143.

Weete, J. D., Abril, M., \& Blackwell, M. (2010). Phylogenetic distribution of fungal sterols. PloS one, 5: e10899. 


\section{Table and Figure legends}

Table 1. Results of ANOVA followed by Tukey's post hoc test, testing the difference of Shannon-Wiener Index applied to fatty acids $\left(\mathrm{H}_{\mathrm{FA}}\right)$ and to sterols $\left(\mathrm{H}_{\mathrm{ST}}\right)$ between the different fractions: ZP (Zoospores), HS (Host+Sporangia) and H (Uninfected host) for diatom and cyanobacterium systems. ND: not determined, as cyanobacteria do not contain sterols. NSS: not statistically significant.

Fig. 1. Cultivation and sample fractionation procedure applied to acquire samples for subsequent fatty acid (FA), sterol (St), and particulate organic carbon (POC) analyses, and to determine prevalence of infection as well as host, bacteria (B), sporangia and zoospore (ZP) densities.

Fig. 2. Fatty acid content (A) and composition (B, C, D) of different fractions in the diatom and cyanobacterium systems. A: Values are given as means of replicates \pm standard deviation (SD). B: Fatty acids are grouped into polyunsaturated (PUFA), monounsaturated (MUFA), saturated (SAFA), branched, and unidentified fatty acids. Numbers reported on the bars refer to mean of absolute concentrations of the respective fatty acid group $\left(\mu \mathrm{g} \mathrm{mgC}^{-1}\right) . \mathrm{C}, \mathrm{D}$ : Fatty acid composition of Zoospores and Uninfected hosts for the diatom (C) and cyanobacterium (D) systems. Values are given as means of replicates \pm SD

Fig. 3. Sterol contents (A) and composition (B) of different fractions in the diatom and cyanobacterium systems. A: Values are given as means \pm standard deviation of replicates. B: Other sterols: traces of lanosterol and three other unidentified sterols. Numbers refer to mean of absolute concentrations of each sterol $\left(10^{2} \mathrm{ng} \mathrm{mgC}^{-1}\right)$. ND: Not detected. 


\section{Supplemental Figures and Tables legends}

FIGURE S1 Shannon-Wiener Index applied to fatty acids $\left(\mathrm{H}_{\mathrm{FA}}, \mathrm{A}\right)$ and to sterols $\left(\mathrm{H}_{\mathrm{ST}}, \mathrm{B}\right)$ for the different fractions of diatom and cyanobacterium systems. Values are given as means of replicates \pm standard deviation. ND: Not detected

\section{TABLE S1}

Fatty acid composition and content of infected and uninfected host $A$. formosa (A) or $P$. agardhii (B) and of CCAP4086/1 (A. formosa -infecting chytrid) (A) and Chy-Kol2008 (P. agardhii -infecting chytrid) (B) zoospores. (SAFA: saturated fatty acid, MUFA: monounsaturated fatty acid, PUFA: polyunsaturated fatty acids). Values are given in percentages as means of replicates \pm standard deviation. nd: not detected. The 27 fatty acids which represent at least $1 \%$ of total FA in at least one fraction of the two systems are colored in grey. 
Fig1

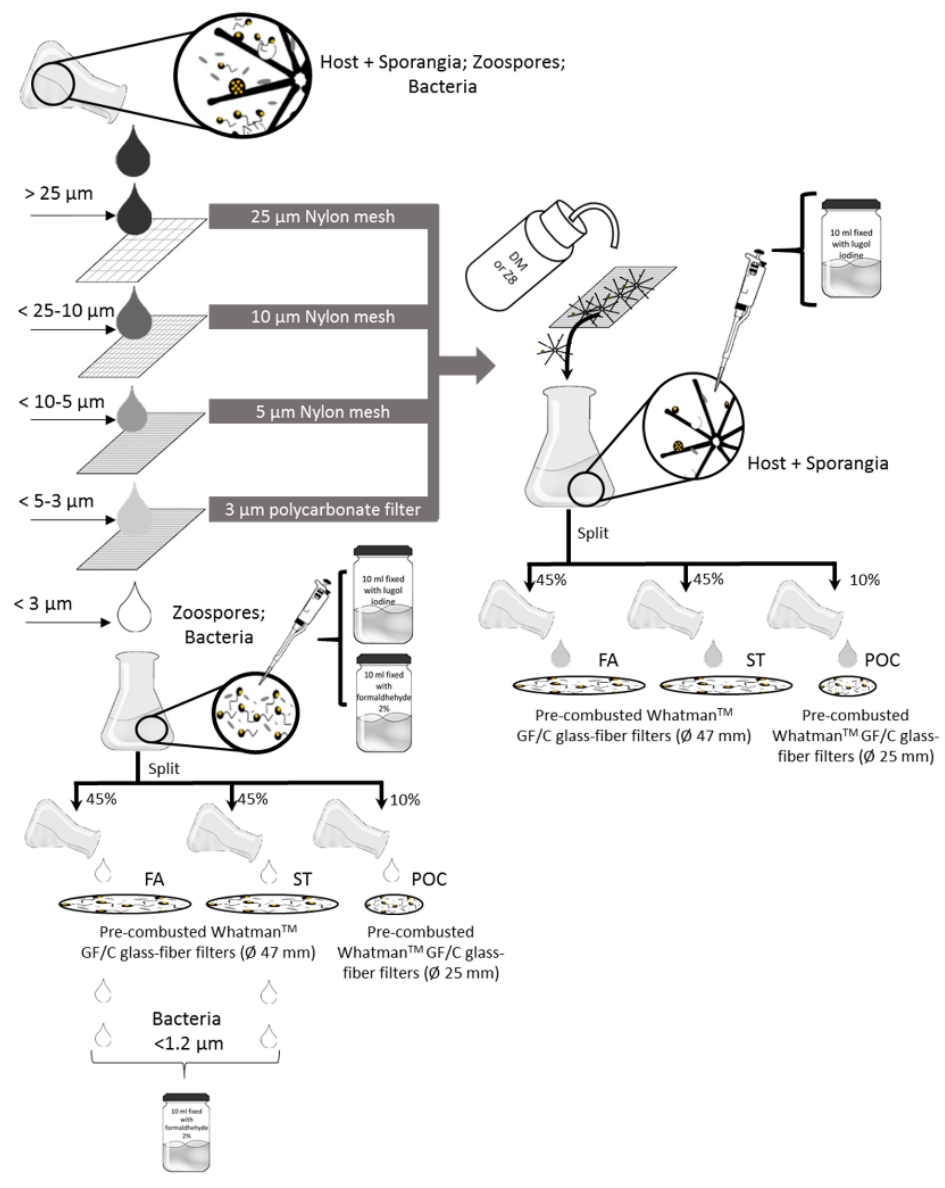


Fig 2
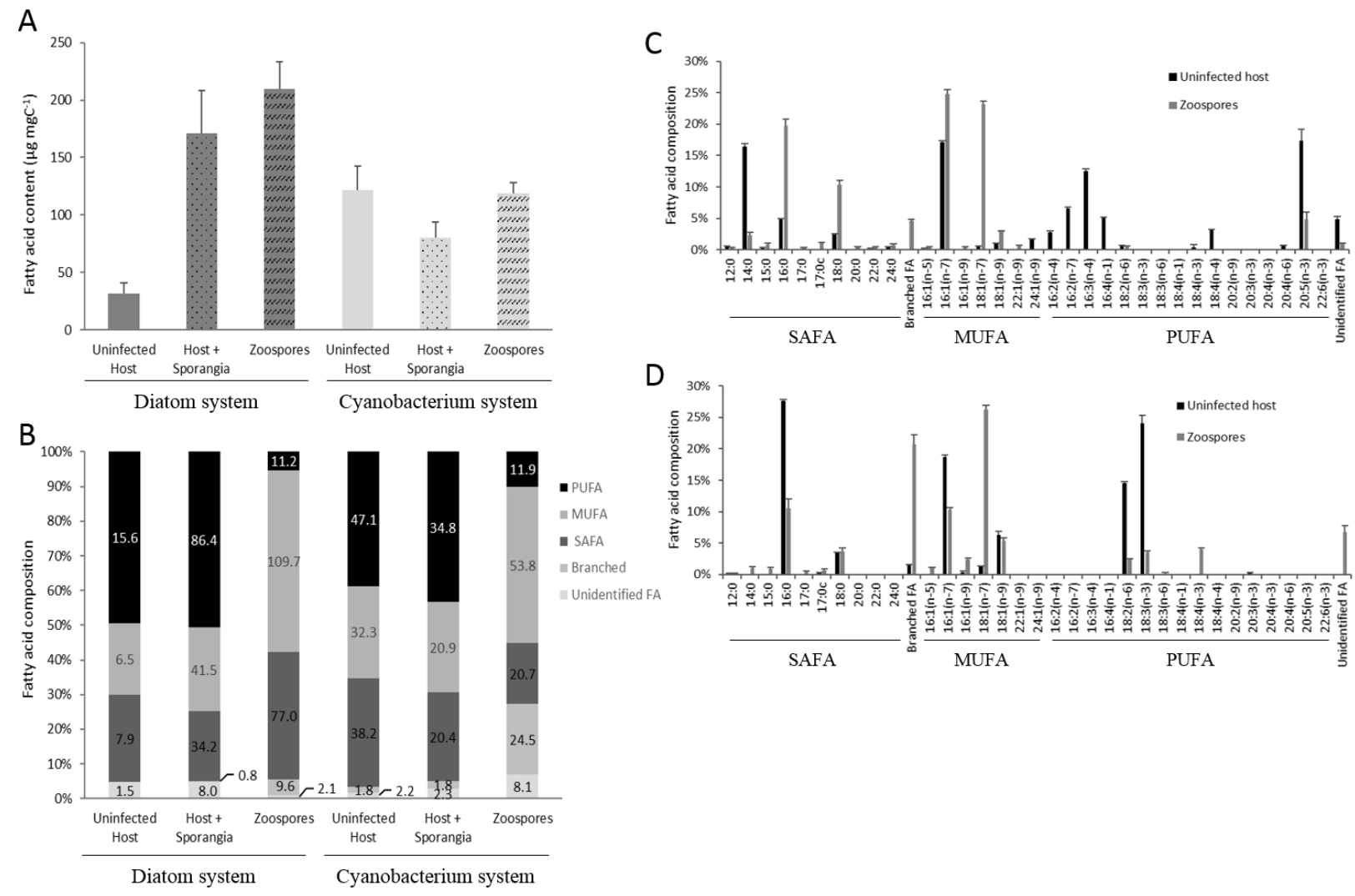
Fig 3

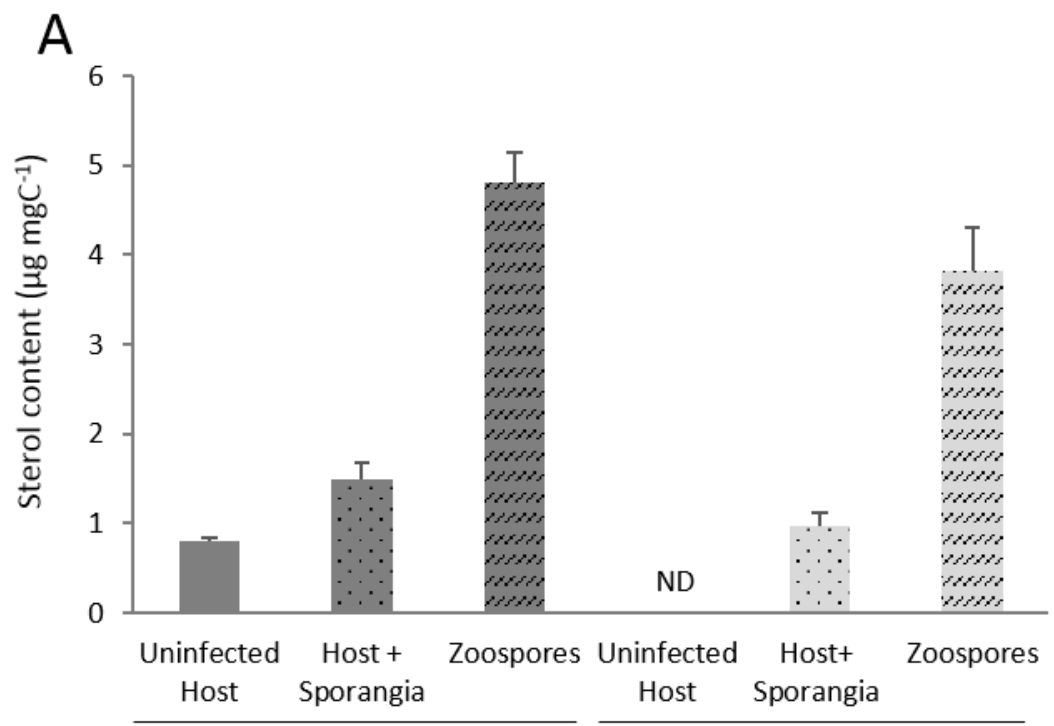

B

Diatom system

Cyanobacterium system

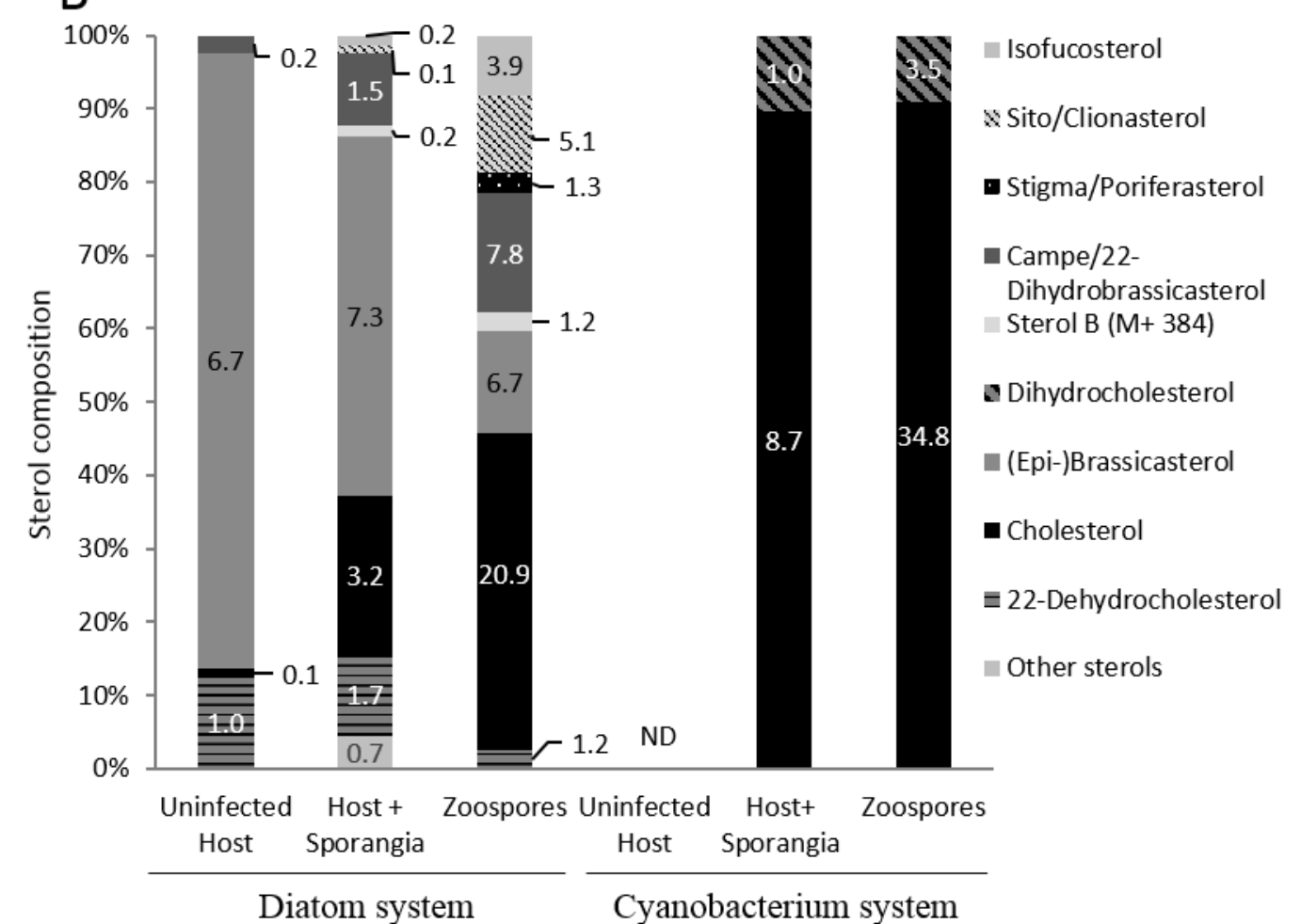


Table 1

Fractions

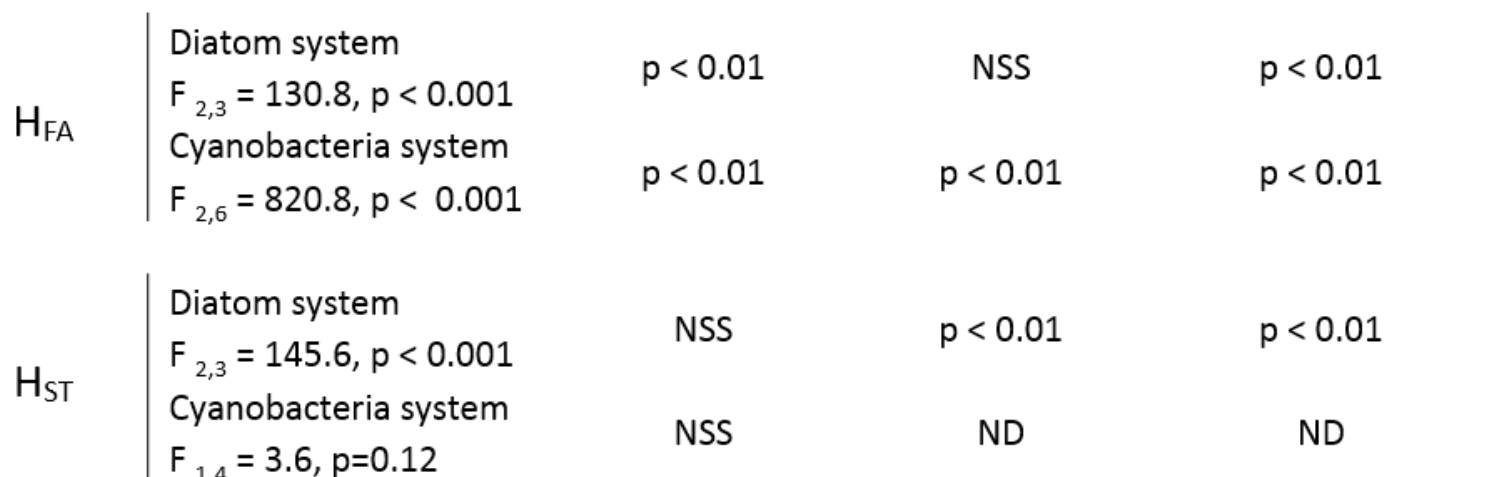




\section{Supporting Information}

Comparison of sterol and fatty acid profiles of chytrids and their hosts reveals trophic upgrading of nutritionally inadequate phytoplankton by fungal parasites

Mélanie Gerphagnon ${ }^{1, a, *}$, Ramsy Agha ${ }^{1 \mathrm{a}}$, Dominik Martin-Creuzburg ${ }^{2}$, Alexandre Bec ${ }^{3}$, Fanny Perriere $^{3}$, Cecilia Rad-Menéndez ${ }^{4}$, Claire M.M. Gachon ${ }^{4}$, and Justyna Wolinska ${ }^{1,5}$

FIGURE S1 Shannon-Wiener Index applied to fatty acids ( $\left.\mathrm{H}_{\mathrm{FA}}, \mathrm{A}\right)$ and to sterols $\left(\mathrm{H}_{\mathrm{ST}}, \mathrm{B}\right)$ for the different fractions of diatom and cyanobacterium systems. Values are given as means of replicates \pm standard deviation. ND: Not detected

TABLE S1. Fatty acid composition and content of infected and uninfected host A. formosa (A) or P. agardhii (B) and of CCAP4086/1 (A. formosa - infecting chytrid) (A) and Chy-Kol2008 (P. agardhii - infecting chytrid) (B) zoospores. (SAFA: saturated fatty acid, MUFA: monounsaturated fatty acid, PUFA: polyunsaturated fatty acids). Values are given in percentages as means of replicates \pm standard deviation. nd: not detected. The 27 fatty acids which represent at least $1 \%$ of total FA in at least one fraction of the two systems are colored in grey. 


\section{FIGURE S1}
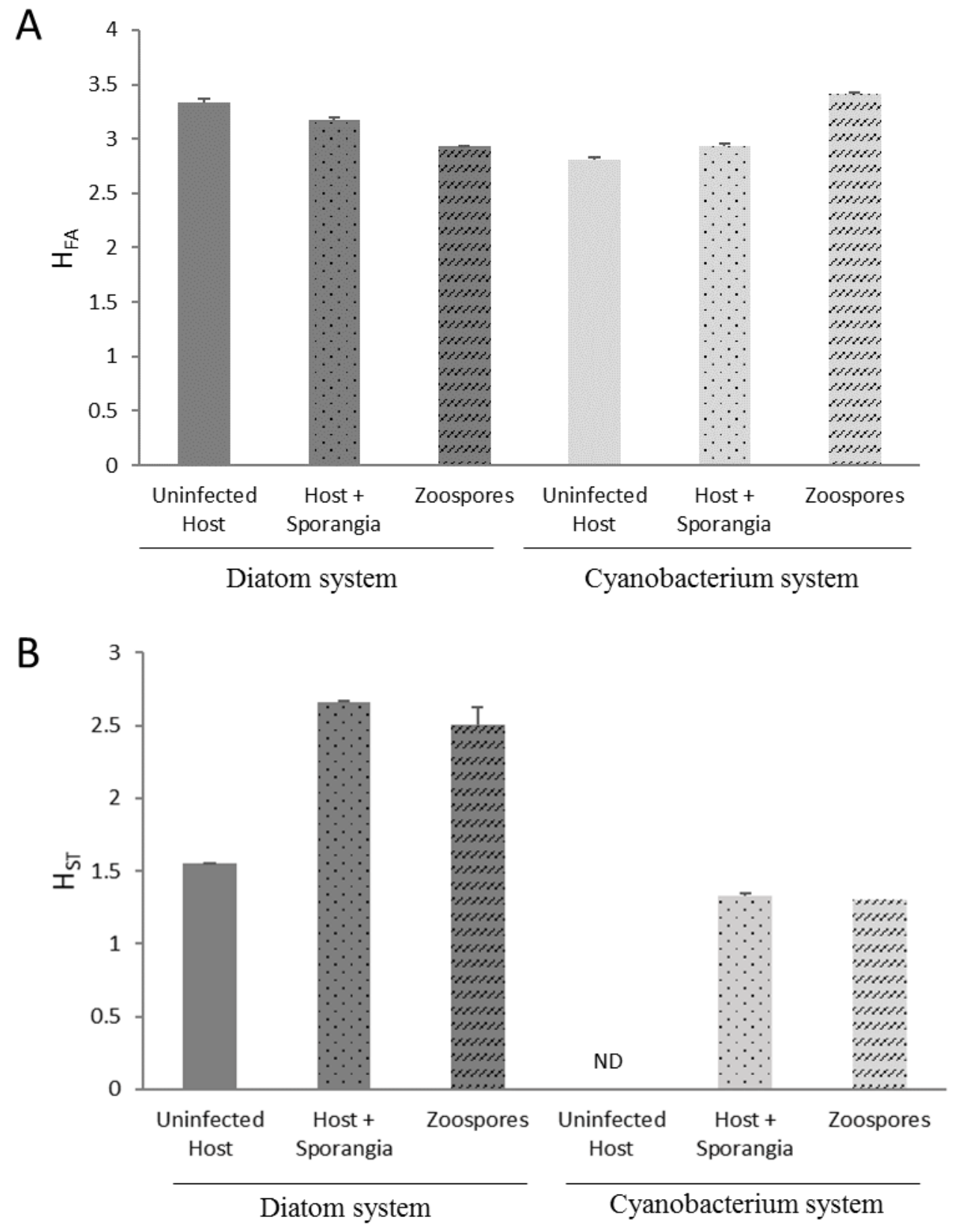


\section{Table S1}

2

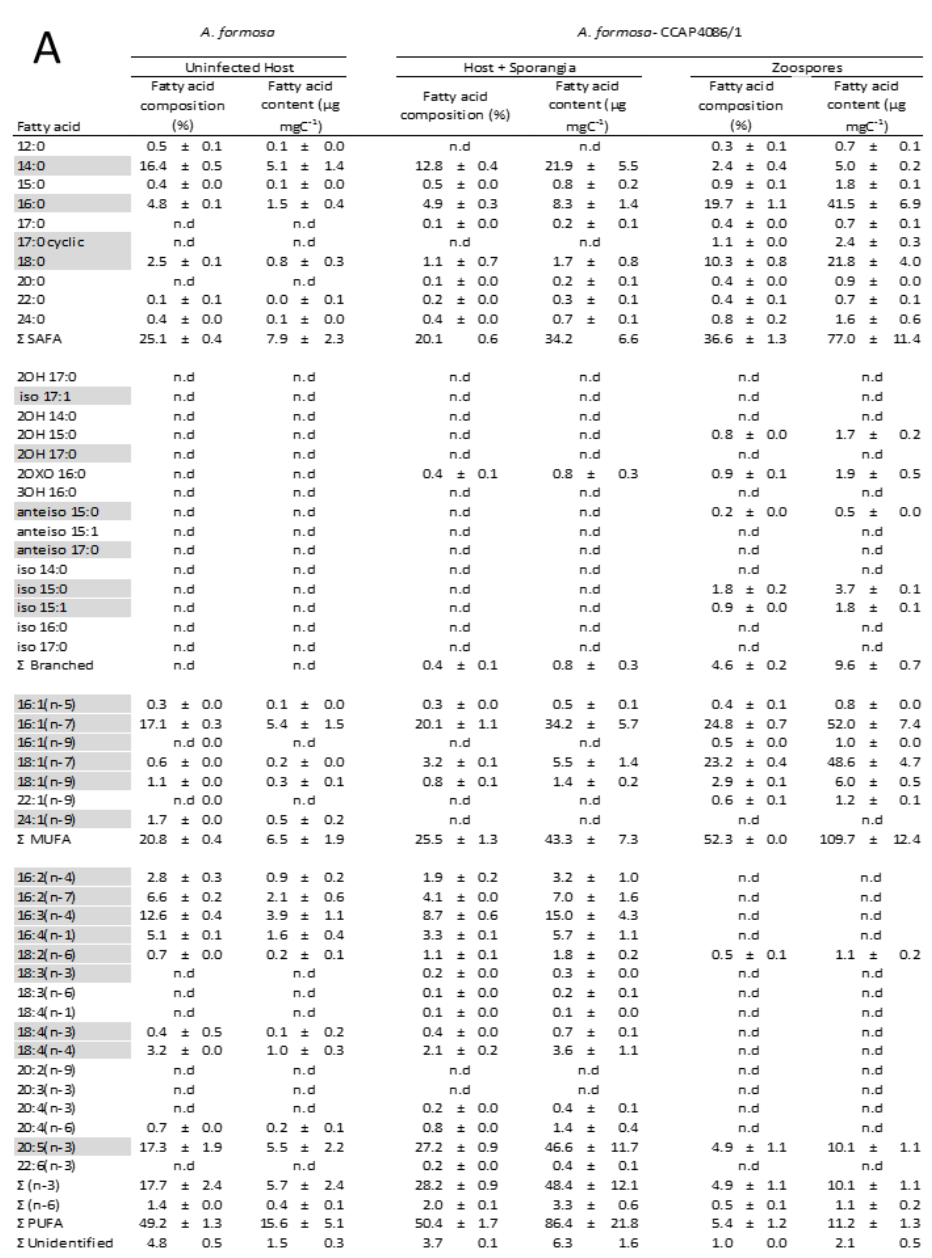

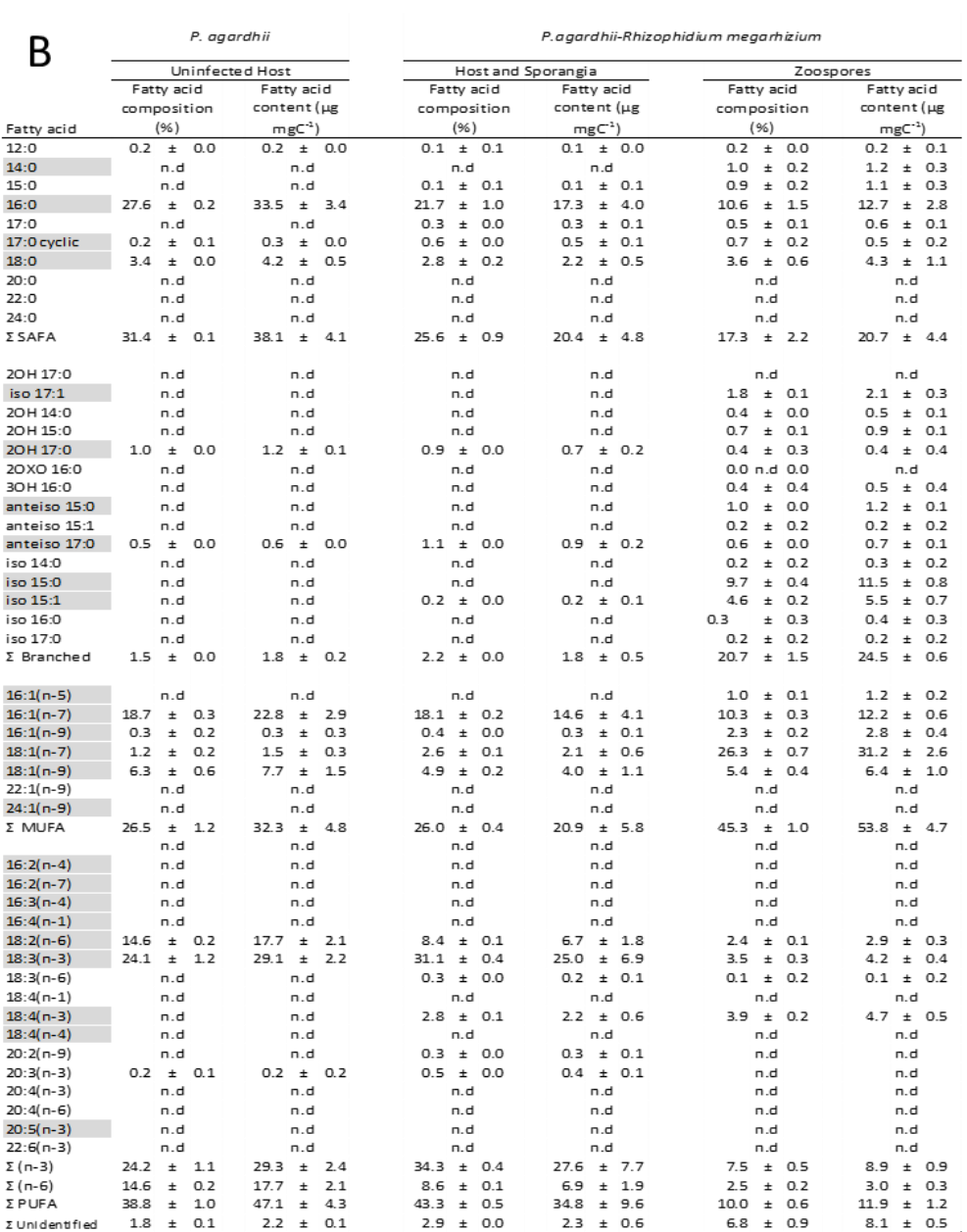


